\title{
Malignant Transformation from Endometriosis to Atypical Endometriosis and Finally to Endometrioid Adenocarcinoma within 10 Years
}

\author{
Yasuhito Tanase $^{a}$ Naoto Furukawa ${ }^{a}$ Hiroshi Kobayashia \\ Takashi Matsumoto ${ }^{\mathrm{b}}$ \\ ${ }^{a}$ Department of Obstetrics and Gynecology, Nara Medical University, Kashihara, and \\ ${ }^{b}$ Department of Gynecology, Osaka Central Hospital, Osaka, Japan
}

\section{Key Words}

Atypical endometriosis · Malignant transformation · Endometriosis

\begin{abstract}
Atypical endometriosis is reported to possess a precancerous potential attributed to premalignant changes characterized by cytological atypia and architecture proliferation. Moreover, the coexistence of atypical endometriosis and neoplasms had been reported. However, cases of atypical endometriosis transformation to carcinoma are rarely reported. We describe the case of a 33-year-old woman who had a long therapeutic history of endometriosis. Three laparoscopic surgeries were performed to treat endometriosis. After the third surgery, she was diagnosed as having grade 1 endometrioid adenocarcinoma. The histological review of the previous surgery confirmed the diagnosis of atypical endometriosis based on the second specimen. The patient's disease progressed from a benign endometriotic cyst to atypical endometriosis and finally to endometrioid adenocarcinoma within 10 years. When we encounter cases of atypical endometriosis, it is necessary to consider the possibility of ovarian cancer and carefully follow the patients for long periods.
\end{abstract}


Tanase et al.: Malignant Transformation from Endometriosis to Atypical Endometriosis and Finally to Endometrioid Adenocarcinoma within 10 Years

\section{Case Report}

A 33-year-old woman (gravida 0, para 0) was referred to Osaka Central Hospital (Osaka, Japan) for gynecological, fertility-preserving laparoscopic surgery. According to her surgical history, two prior laparoscopic surgeries had been performed to treat endometriosis. At the age of 24, she underwent her first laparoscopic cystectomy for left ovarian endometrioma at a community hospital. When she was 29 years old, a second laparoscopic surgery (laparoscopic cystectomy for a right ovarian cyst) was performed at the same institution. The histological examinations of both specimens revealed benign endometriotic cysts. However, approximately 7 months following the second surgery, ultrasonographic and pelvic examinations revealed bilateral ovarian cysts. In response, we administered gonadotropinreleasing hormone agonist therapy (buserelin acetate, $900 \mu \mathrm{g} /$ day) every 6 months. Upon termination of treatment, the cysts gradually enlarged. At 33 years of age, our patient got married. She desired children and was therefore referred to our institution for fertility treatment via laparoscopic surgery.

Upon presentation to our hospital, both her ovaries were enlarged (left, $3 \mathrm{~cm}$; right, 4 $\mathrm{cm}$ ) because of endometrioma. MRI and ultrasound scans showed no solid component within the bilateral cysts. Initially, we considered terminating the hormonal therapy to allow for natural pregnancy. However, she did not become pregnant during a follow-up period of approximately 5 months. Therefore, our patient consented to a third laparoscopic procedure to improve her fertility.

A preoperative MRI scan revealed a solid 2-cm component of her right ovarian endometrioma. Although the imaging was not enhanced, the possibility of malignancy could not be overlooked. Therefore, we recommended exploratory laparotomy to be performed at another institution. However, the patient and her husband rejected this recommendation and strongly insisted upon a third laparoscopic surgery at our institution. Our patient was fully informed of the treatment options and submitted written consent after which we agreed to perform the third laparoscopic surgery.

Notably, the third laparoscopic surgery was performed at our institution approximately 10 years after the first surgery. The patient's cancer antigen 125 serum level was within the normal range and both ovaries, which were strongly adhered to surrounding tissues, were enlarged (right, $5 \mathrm{~cm}$; left, $4 \mathrm{~cm}$ ). The cul-de-sac was obliterated with dense pelvic adhesions. Laparoscopic cystectomy for both ovarian cysts and adhesiolysis were performed.

The patient had an uneventful recovery. A histological examination revealed a transition between the endometrioid adenocarcinoma and the directly adjacent endometriosis (fig. 1). The cytological result of the small amount of ascites was negative. We reviewed the external slides of the previous two surgeries and confirmed the diagnosis of atypical endometriosis based on the second specimen (fig. 2). The patient was diagnosed as having FIGO stage 1c(2), grade 1 ovarian endometrioid adenocarcinoma. Once informed of our diagnosis, our patient desired to attempt to preserve her fertility and rejected complete curative surgery for ovarian cancer.

For the fourth surgery, we performed laparoscopic salpingo-oophorectomy of the right ovary, biopsy of the left ovary and partial omentectomy. All specimens showed no pathological malignancy. Postoperatively, the patient underwent three courses of chemotherapy consisting of $175 \mathrm{mg} / \mathrm{m}^{2}$ of paclitaxel and carboplatin (AUC 5) every 3 weeks. A follow-up examination conducted 7 years after the fourth surgery showed no evidence of recurrence. 
Tanase et al.: Malignant Transformation from Endometriosis to Atypical Endometriosis and Finally to Endometrioid Adenocarcinoma within 10 Years

\section{Discussion}

Our patient presented with recurrent endometriosis, and within 10 years, her disease progressed from benign to atypical endometriosis and finally to endometrioid adenocarcinoma through three laparoscopic surgeries. In 1925, Sampson [1] first described malignant changes in endometriosis and proposed the following criteria for diagnosing the carcinomatous development in endometriosis: (i) coexistence of carcinoma and endometriosis within the same ovary, (ii) a similar histological pattern and (iii) exclusion of a second malignant tumor elsewhere. Later, in 1953, Scott [2] postulated that in addition to the criteria by Sampson, morphological changes demonstrated by benign endometriosis that are contiguous with malignant tissue is a prerequisite for adjudication of a malignancy originating from endometriosis. In the present case, the diagnosis of atypical endometriosis was made based on histopathological criteria reported by LaGrenade and Silverberg [3] and Czernobilsky and Morris [4], which include features of eosinophilic cytoplasm, large hyperchromatic or pale nuclei with moderate to marked pleomorphism, an increased nuclear to cytoplasmic ratio, cellular crowding and stratification or tufting. The present case fulfilled all of these criteria, and a transition between endometriosis and endometrioid adenocarcinoma was observed. Accordingly, we diagnosed our case as a malignant transformation of endometriosis through atypical endometriosis.

Although the pathogenesis of malignant changes in endometriosis remains unclear, some studies suggest that certain aspects of endometriosis are similar to those of other malignancies [5]. There are numerous reported cases of malignancies arising from endometriotic deposits and substantial histological evidence that endometriosis is associated with ovarian cancer, particularly endometrioid carcinoma and clear-cell carcinoma [6-9].

Atypical endometriosis is reported to possess precancerous potential attributed to premalignant changes characterized by cytological atypia and architecture proliferation. However, atypical endometriosis is considered as a reactive change to inflammation. Mild dysplasia of the uterine cervix is considered a result of inflammation, and mild atypical endometriosis may be reactive to local severe inflammation and/or superficial ulceration with regenerative activity. A study by Ogawa et al. [9] on ovarian endometriosis associated with ovarian carcinoma reported that the correlation between inflammation and atypical epithelia in endometriosis should be evaluated considering the influence of severe stromal inflammation. In addition, Czernobilsky and Morris [4] described that mild atypism was usually associated with severe stromal inflammation as well as areas of epithelial denudation and regeneration. However, these studies also concluded that most cases of atypical endometriosis are not a result of reactive inflammation but rather possess a precancerous potential for ovarian carcinoma because epithelial atypia occur irrespective of severe stromal inflammation. Reportedly, atypical endometriosis possesses a precancerous potential, and numerous studies have described malignant transformation in endometriosis. Moreover, the coexistence of endometriosis and neoplasms has been described. However, cases of atypical endometriosis transformation to carcinoma are rarely reported.

Czernobilsky and Morris [4] report the incidence of atypical endometriosis without neoplasm to be $3.6 \%$ (7/194), Fukunaga et al. [10] report it to be $1.7 \%(4 / 255)$, Seidmann [11] reports it to be $32.3 \%$ (34/105) and Bayramoglu and Duzcan [12] report it to be $5.8 \%$ $(7 / 120)$. In contrast, the incidence of atypical endometriosis with neoplasm was reported as follows: $22.8 \%$ (29/127) by Ogawa et al. [9], 14.7\% (33/224) by Fukunaga et al. [10] and $4.4 \%(8 / 183)$ by Oral et al. [13]. These studies agreed that the so-called 'atypical endometri- 
Tanase et al.: Malignant Transformation from Endometriosis to Atypical Endometriosis and Finally to Endometrioid Adenocarcinoma within 10 Years

osis' may represent a step in the carcinogenic pathway. However, factors leading up to atypia remain poorly understood.

In the literature, the incidence of atypical endometriosis in ovarian cancer reportedly ranges from 4.4 to $22.8 \%$ and without neoplasm involvement from 1.7 to $32.3 \%$. A subset of atypical endometriosis is considered to possess the capacity to transform into ovarian carcinoma. However, when we encounter cases of atypical endometriosis, it is necessary to consider the possibility of ovarian cancer and carefully follow these cases. Nevertheless, the issue of how to conduct a follow-up of women who underwent surgery for endometriosis and for whom the final diagnosis was atypical endometriosis remains. This is because there are no guidelines regarding the postoperative monitoring of these women, such as counseling for hormonal therapy and luteal hormonal therapy, oral contraceptives or the role of definitive total hysterectomy and bilateral salpingo-oophorectomy following conservative primary surgery.

Moll et al. [14] reported a chronological association between ovarian endometriosis showing atypical foci and subsequent large clear-cell carcinoma arising in the same ovary 3 years later. Their case was the first documented description of atypical endometriosis that underwent complete malignant transformation within 3 years and subsequently gave rise to invasive clear-cell carcinoma. Two patients reported by Hyman [15] developed extragonadal endometrioid carcinoma 7 and 17 years, respectively, after bilateral salpingo-oophorectomy for focally atypical endometriosis. In a follow-up study by Fukunaga et al. [10], 3 of 4 patients with atypical endometriosis did not develop malignant epithelial tumors, although the fourth patient developed endometrioid carcinoma in the abdominal wall 18 months after left oophorectomy, with an average overall survival period for all patients of 2.5 years (range, 1.5-3.5). In comparison, in a study by Seidman [11], 1 of 20 patients with either complex or atypical hyperplasia developed clinically evident endometrioid adenocarcinoma within a follow-up period of 8.6 years. Furthermore, Modesitt et al. [7] reported a case of carcinosarcoma arising from atypical endometriosis that developed within 16 years because of the cesarean scar. In the present case, disease progressed from an endometriotic cyst to atypical endometriosis and finally to endometrioid adenocarcinoma within 10 years over a span of three surgeries.

\section{References}

1 Sampson JA: Endometrial carcinoma of the ovary arising in endometrial tissue in that organ. Arch Surg 1925;10:1-72.

2 Scott RB: Malignant changes in endometriosis. Obstet Gynecol 1953;2:283-289.

3 LaGrenade A, Silverberg SG: Ovarian tumors associated with atypical endometriosis. Hum Pathol 1988;19:1080-1084.

4 Czernobilsky B, Morris WJ: A histologic study of ovarian endometriosis with emphasis on hyperplastic and atypical changes. Obstet Gynecol 1978;53:318-323.

5 Helen S, William C, Stephane L: Endometriosis-associated ovarian cancer: a clinicopathologic review. J Obstet Gynaecol Can 2004;26:709-715.

6 Tagashira Y, Shimada M, Kigawa J, Iba T, Terakawa N: Ovarian endometrioid adenocarcinoma arising from endometriosis in a young woman. Gynecol Oncol 2003;91:643-647.

7 Modesitt SC, Tortolaro-Luna G, Robinson JB, Jubilee B, Gershenson DM, Wolf JK: Ovarian and extraovarian endometriosis-associated cancer. Obstet Gynecol 2002;100:788-795.

8 Vercellini P, Scarfone G, Bolis G, Carinelli S, Crosiqnani PG: Site of origin of epithelial ovarian cancer: the endometriosis connection. Br J Obstet Gynaecol 2000;107:1155-1157.

9 Ogawa S, Kaku T, Amada S, Kobayashi T, Hirakawa T, Ariyoshi K, Kamura T, Nakano H: Ovarian endometriosis associated with ovarian carcinoma: a cliniclpathological and immunohistochemical study. Gynecol Oncol 2000;77:298-304.

10 Fukunaga M, Nomura K, Ishikawa E, Ushigome S: Ovarian atypical endometriosis: its close association with malignant epithelial tumors. Histopathology 1997;30:249-255. 


\section{Case Reports in Oncology}

\begin{tabular}{l|l}
\hline Case Rep Oncol 2013;6:480-484 & \\
\hline DOI: 10.1159/000355282 & $\begin{array}{l}\text { ○ 2013 S. Karger AG, Basel } \\
\text { www.karger.com/cro }\end{array}$ \\
\hline
\end{tabular}

Tanase et al.: Malignant Transformation from Endometriosis to Atypical Endometriosis and Finally to Endometrioid Adenocarcinoma within 10 Years

11 Seidman JD: Prognostic importance of hyperplasia and atypia in endometriosis. Int J Gynecol Pathol 1996;15:1-9.

12 Bayramoglu H, Duzcan E: Atypical epithelial change and mutant p53 gene expression in ovarian endometriosis. Pathol Oncol Res 2001;7:33-38.

13 Oral E, IIvan S, Tustas E, Korbeyli B, Bese T, Demirkiran F, Arvas M, Kosebay D: Prevalence of endometriosis in malignant epithelial ovary tumours. Eur J Obstet Gynecol Reprod Biol 2003;109:97-101.

14 Moll UM, Chumas JC, Chalas E, Mann WF: Ovarian carcinoma arising in atypical endometriosis. Obstet Gyneycol 1990;75:537-539.

15 Hyman MP: Extraovarian endometrioid carcinoma: a review of the literature and report of two cases with unusual features. Am J Clin Pathol 1977;68:522-527.
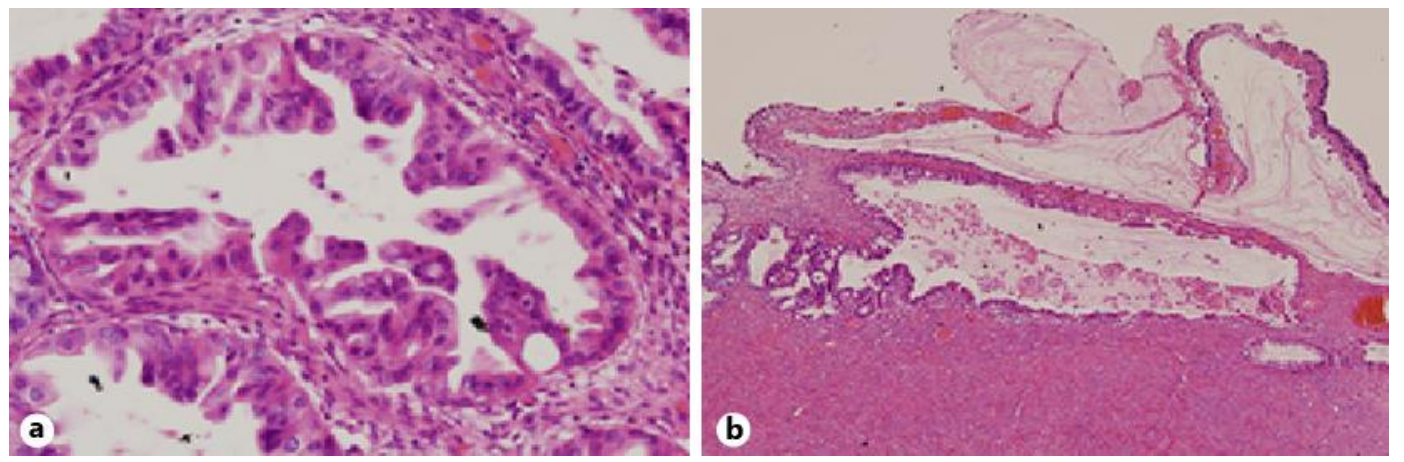

Fig. 1. Pathological finding of the third specimen for her left ovarian cyst (H\&E staining; a $\times 40, \mathbf{b} \times 400$ ). a Grade 1 endometrioid adenocarcinoma was observed. $\mathbf{b}$ The transition between the carcinoma and the benign endometriosis was detected.

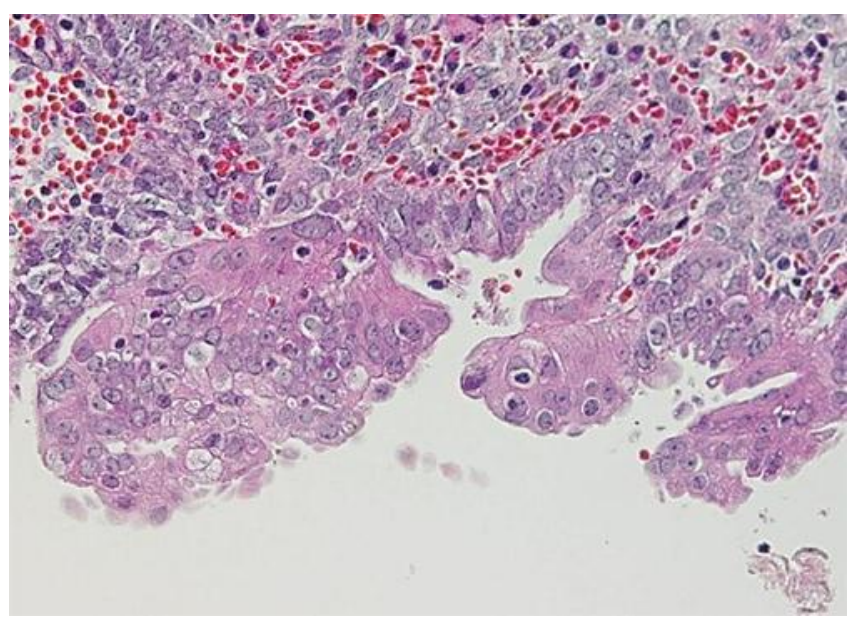

Fig. 2. Pathological finding of the second specimen for her right ovarian cyst (H\&E staining; $\times 400)$. Atypical features were observed, including eosinophilic cytoplasm, large hyperchromatic or pale nuclei with moderate pleomorphism, an increased nuclear to cytoplasmic ratio, cellular crowding and stratification. 